\title{
Contourlet and Gould Transforms for Hybrid Image Watermarking in RGB Color Images
}

\author{
Reena Thomas ${ }^{1, *}$ and M. Sucharitha ${ }^{2}$ \\ ${ }^{1}$ Department of ECE, Noorul Islam Centre for Higher Education, Kumaracoil, 629180, India \\ ${ }^{2}$ Department of ECE, Malla Reddy College of Engineering and Technology, Hyderabad, 500001, India \\ *Corresponding Author: Reena Thomas. Email: reenathomaspaper12@gmail.com \\ Received: 02 October 2021; Accepted: 03 November 2021
}

\begin{abstract}
The major intention of this work is to introduce a novel hybrid image watermarking technique for RGB color images. This hybrid watermarking algorithm uses two transforms such as Contourlet and Gould transform. The Contourlet transform is used as first stage while the Gould transform is used as second stage. In the watermark embedding phase, the R, G and B channels are transformed using Contourlet transform. The bandpass directional sub band coefficients of Contourlet transformed image are then divided into $2 \times 2$ sub-blocks. The sub-blocks are then transformed using Gould transform and the watermark information is embedded on the initial coefficients of each sub-blocks. After the embedding of watermark, inverse of Gould and Contourlet are taken to obtain the watermarked image. The reverse operation is performed to extract the watermark. Results obtained from attack-based experimentation for checking robustness implicate the superiority of this method in comparison with conventional methods. Better values are obtained for Embedding Rate, Structural Similarity Index Metric (SSIM) and Peak Signal to Noise Ratio (PSNR).
\end{abstract}

Keywords: Contourlet transform; embedding rate; Gould transform; hybrid image watermarking; robustness

\section{Introduction}

Image watermarking [1-3] is a technique where information is hidden on a host image. These watermarking techniques have applications in protecting and verifying the copyright of host image. Today, because to the fast advancement in digital technologies, especially the internet, huge amount of media such as image are transmitted and shared around the world. Protecting and verifying the ownership of the image is a challenging task. Watermarking is an advance solution to ensure the copyright of images. Watermarking system is categorized on the basis of watermark visibility. The watermark will be transparent in a visible watermarking while it is not transparent in an invisible watermarking technique [4-6].

Watermarking can be performed on spatial domain [7-9] as well as frequency domain [10-12]. In spatial domain method, secret data is directly induced on pixel intensities without any transformation in host image

This work is licensed under a Creative Commons Attribution 4.0 International License, which permits unrestricted use, distribution, and reproduction in any medium, provided the original work is properly cited. 
pixel intensities. Since the pixel intensities are easily modifiable, these techniques are highly vulnerable to attacks. In a frequency domain scheme, the watermark is induced on the host after applying a transform. These frequency domain methods are more resistant to attacks when compared to spatial domain scheme, because of its less sensitivity to such attacks.

Many researchers are working on image watermarking algorithm $[13,14]$ to improve the performance such as embedding rate, image quality and robustness. The term robustness addresses the capability to recover the watermark even though the image is subjected to various types of spatial attacks. The spatial domain schemes include Histogram Shift [15], Differential Expansion [16] and Predictive Expansion [17]. Frequency domain schemes include transforms such as Discrete Fourier Transform (DFT) [18], Discrete Cosine Transform (DCT) [19] and Discrete Wavelet Transform (DWT) [20]. These transform also uses the decomposition method such as Singular Value Decomposition (SVD) [21], QR Decomposition, Schur Decomposition [22] and LU Decomposition [23].

\section{Overview of Transforms}

\subsection{Contourlet Transform}

Initially, the Contourlet transform [24] was proposed using multiscale filtering and block ridgelet transform that works on continuous domain. Later the ridgelet transform was replaced by frequency partitioning and the transform was named as second generation curvelet transform. The Contourlet transform needs an edge detection process and adaptive representation. This Contourlet uses a double filter bank to obtain the sparse representation for smooth contour images. It performs the edge detection process using a transform similar to wavelet. For detecting the Contour segment, it uses a local directional transform.

The multiscale decomposition can be achieved by using Laplacian Pyramid (LP). At each level of Laplacian Pyramid decomposition, a low pass of the original image is generated. In multidirectional filter bank the down sampling the signal $x[n]$ is represented as $x[M n]$. Here $M$ is a matrix with integer values. Fig. 1 shows the Countourlet transform of a test image, where it is decomposed into two pyramid levels.

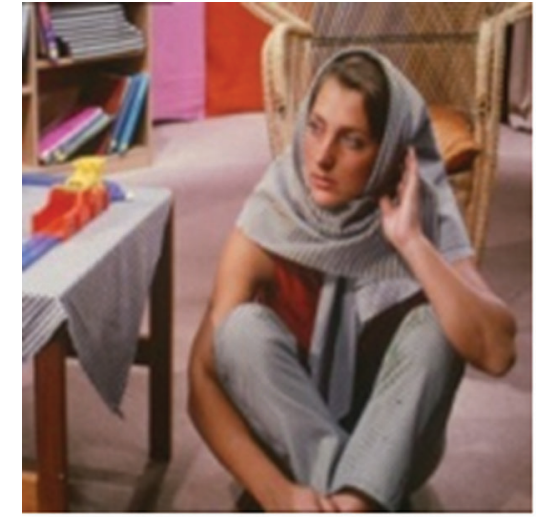

(a)

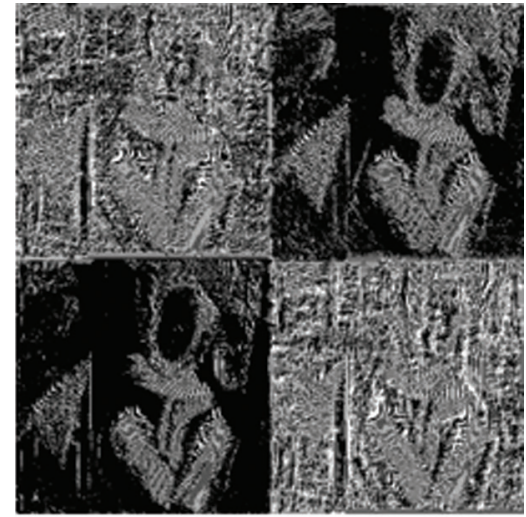

(b)

Figure 1: Frequency transformation (a) Barbara image, (b) pyramidal levels

\subsection{Gould Transform}

In 2006, Le et al. proposed discrete Gould transform [25]. In this 2D Gould transform a matrix or image $f$ having a size $N \times N$ can be transformed to $F$. This transform can be performed using a Gould transform 
matrix represented as $G$. The $2 \times 2$ representation of $G$ can be written as,

$G=\left[\begin{array}{cc}1 & 0 \\ -1 & 1\end{array}\right]$

Therefore, the 2D Gould transform for a 2D gray scale image $f$ can be represented as.

$F=G f G^{T}$

where $G^{T}$ is the transpose of the transform matrix $G$. Since the Gould transform matrix is having the size of $2 \times 2$ the image $f$ must be subdivided into $2 \times 2$ subblocks. Therefore, the transformed image $F$ also has the size of $2 \times 2$ which is represented as,

$F=\left[\begin{array}{cc}1 & 0 \\ -1 & 1\end{array}\right]\left[\begin{array}{ll}f_{11} & f_{12} \\ f_{21} & f_{22}\end{array}\right]\left[\begin{array}{cc}1 & -1 \\ 0 & 1\end{array}\right]$

$F=\left[\begin{array}{cc}f_{11} & f_{12}-f_{11} \\ f_{21}-f_{11} & f_{22}+f_{11}-f_{12}-f_{21}\end{array}\right]$

Let $f$ ij be the pixel in a $2 \times 2$ subimage in ith row and jth column. In a $2 \times 2$ subimage the discrete Gould transform coefficients specifies the differencing between the neighbouring pixels. The inverse discrete Gould transform for the transformed image F can be obtained using the relation,

$f=G^{-1} F\left[G^{T}\right]^{-1}$

$f=\left[\begin{array}{cc}F_{11} & F_{11}+F_{12} \\ F_{11}+F_{21} & F_{11}+F_{12}+F_{21}+F_{22}\end{array}\right]$

From Eq. (6), if all the Gould coefficients are used for embedding (i.e., all the coefficients are incremented by 1 , then the maximum increase in pixel intensity after taking inverse Gould transform will be increased by 4 . Similarly, if all the Gould coefficients are decremented by 1 , then the decrease in pixel intensity after taking inverse will be decremented by 4 .

\section{Proposed Hybrid Watermarking Algorithm}

\subsection{Watermark Embedding}

Fig. 2 depicts a detailed flow of embedding process. Let $I(x, y, z)$ be the RGB host image, $I_{R}(x, y)$ represents the red channel, $I_{G}(x, y)$ represents the green channel and $I_{B}(x, y)$ represents the blue channel. First step is to obtain the Contourlet transform for R, G and B channels followed by selecting the subband coefficients of Contourlet transform for embedding the watermark. Let $f_{R}(x, y)$ represents the red component bandpass directional sub-band Contourlet coefficients. Similarly, $f_{G}(x, y)$ and $f_{B}(x, y)$ represents the green and blue component contourlet bandpass directional sub-band coefficients for embedding. Sub-divide these coefficients into $2 \times 2$ subblocks. Let $f_{r}(x, y), f_{g}(x, y)$ and $f_{b}(x, y)$ represents the $2 \times 2$ subblocks of the Contourlet coefficients. By taking Gould transform for the $2 \times 2$ subblocks $f_{r}(x, y), f_{g}(x, y)$ and $f_{b}(x, y)$, we get the Gould coefficients as $F_{r}(x, y), F_{g}(x, y)$ and $F_{b}(x, y)$. Let $b_{i}$ represents the watermark bit to be embedded on $2 \times 2$ subblock $F_{r}(x, y)$ where $b_{i} \in[0,1]$. The watermark bit $b_{i}$ can be embedded on $F_{r}(x, y)$ represented by,

$F_{r}(x, y)=\left[\begin{array}{ll}F_{r}(1,1) & F_{r}(1,2) \\ F_{r}(2,1) & F_{r}(2,2)\end{array}\right]$ 


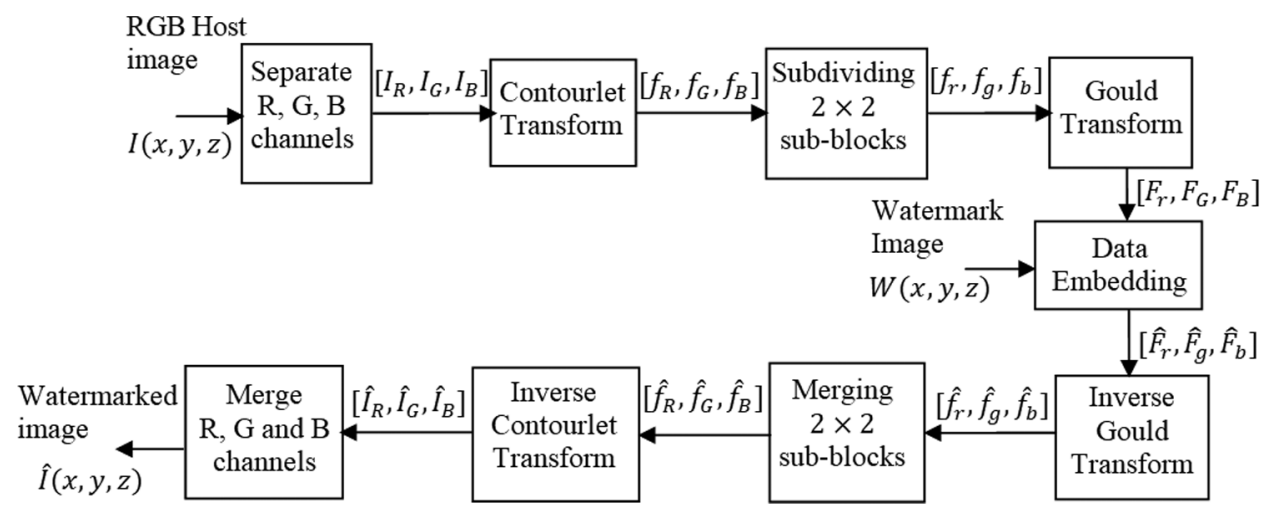

Figure 2: Process flow of proposed watermark embedding scheme

After embedding the watermark bit $b_{i}$ the $2 \times 2$ red Gould coefficients can be modified as,

$\hat{F}_{r}(x, y)=\left[\begin{array}{ll}\hat{F}_{r}(1,1) & F_{r}(1,2) \\ F_{r}(2,1) & F_{r}(2,2)\end{array}\right]$

$\hat{F}_{r}(1,1)=\left\{\begin{array}{l}F_{r}(1,1)-\bmod \left(F_{r}(1,1), \alpha\right)+\left(\frac{\alpha}{2}\right) \text { if } b_{i}=1 \\ F_{r}(1,1)-\bmod \left(F_{r}(1,1), \alpha\right) \text { if } b_{i}=0\end{array}\right.$

Here, $\alpha$ is known as embedding strength. The embedded Gould coefficients $\hat{F}_{r}(x, y)$ is taken inverse Gould transform to obtain the inverse Gould coefficients $\hat{f}_{r}(x, y)$. All the $2 \times 2$ sub-blocks are merged to obtain the complete inverse Gould coefficients values $\hat{f}_{R}(x, y)$. The inverse Gould coefficients are applied with inverse Contourlet coefficients to obtain the marked $\mathrm{R}$ channel $\hat{I}_{R}(x, y)$. The same procedure is applied to other channels to obtain the marked channels $\hat{I}_{G}(x, y)$ and $\hat{I}_{B}(x, y)$. These marked channels are concatenated to obtain the watermarked RGB image $\hat{I}(x, y, z)$. The algorithm for embedding is explained below,

Input: RGB host image $\mathrm{I}(\mathrm{x}, \mathrm{y}, \mathrm{z})$, watermark image $\mathrm{W}(\mathrm{x}, \mathrm{y}, \mathrm{z})$.

Output: Watermarked image $\hat{\mathrm{I}}(\mathrm{x}, \mathrm{y}, \mathrm{z})$

Step 1: Separate the channels from the host image $\mathrm{I}(\mathrm{x}, \mathrm{y}, \mathrm{z})$.

Step 2: Obtain the Contourlet transform for the $R$ channel $I_{R}(x, y)$.

Step 3: Subdivided the bandpass directional subband Contourlet coefficients $\mathrm{f}_{\mathrm{R}}(\mathrm{x}, \mathrm{y})$ to $2 \times 2$ blocks. Let the $2 \times 2$ sub-blocks be $\mathrm{f}_{\mathrm{r}}(\mathrm{x}, \mathrm{y})$.

Step 4: Obtain the Gould transform for the $2 \times 2$ subblocks $\mathrm{f}_{\mathrm{r}}(\mathrm{x}, \mathrm{y})$. Let the transformed Gould coefficients be $\mathrm{F}_{\mathrm{r}}(\mathrm{x}, \mathrm{y})$

Step 5: Embed the watermark data $b_{i}$ on the first coefficient of the $2 \times 2$ subblocks $F_{r}(x, y)$ using Eq. (9). Let the sub-block after embedding will be $\hat{\mathrm{F}}_{\mathrm{r}}(\mathrm{x}, \mathrm{y})$.

Step 6: Obtain the inverse Gould transform for $\hat{\mathrm{F}}_{\mathrm{r}}(\mathrm{x}, \mathrm{y})$. Let the inverse Gould transform be $\hat{\mathrm{f}}_{\mathrm{r}}(\mathrm{x}, \mathrm{y})$

Step 7: Merge the $2 \times 2$ subblocks $\hat{\mathrm{f}}_{\mathrm{r}}(\mathrm{x}, \mathrm{y})$ to obtain $\hat{\mathrm{f}}_{\mathrm{R}}(\mathrm{x}, \mathrm{y})$

Step 8: Obtain the inverse Contourlet transform for $\hat{\mathrm{f}}_{\mathrm{R}}(\mathrm{x}, \mathrm{y})$ to obtain $\hat{\mathrm{I}}_{\mathrm{R}}(\mathrm{x}, \mathrm{y})$.

Step 9: Repeat Step 2 to 8 for $G$ and $B$ channels to obtain $\hat{\mathrm{I}}_{\mathrm{G}}(\mathrm{x}, \mathrm{y})$ and $\hat{\mathrm{I}}_{\mathrm{B}}(\mathrm{x}, \mathrm{y})$. Also merge marked $\mathrm{R}, \mathrm{G}$ and $\mathrm{B}$ channels $\hat{\mathrm{I}}_{\mathrm{R}}(\mathrm{x}, \mathrm{y}), \hat{\mathrm{I}}_{\mathrm{G}}(\mathrm{x}, \mathrm{y})$ and $\hat{\mathrm{I}}_{\mathrm{B}}(\mathrm{x}, \mathrm{y})$ to obtain the watermarked image $\hat{\mathrm{I}}(\mathrm{x}, \mathrm{y}, \mathrm{z})$. 


\subsection{Watermark Extraction}

Fig. 3 depicts a detailed flow of extraction process. Let $I^{\prime}(x, y, z)$ represents the watermarked RGB image. From $I^{\prime}(x, y, z)$, separate the $\mathrm{R}, \mathrm{G}$ and $\mathrm{B}$ channels $I_{R}^{\prime}(x, y), I_{G}^{\prime}(x, y)$ and $I_{B}^{\prime}(x, y)$. Obtain the Contourlet transform for the red channel $I_{R}^{\prime}(x, y)$. Let $f_{R}^{\prime}(x, y)$ represents the bandpass directional sub-band coefficients of the Red channel Contourlet transformed image $I_{R}^{\prime}(x, y)$. Sub-divide the coefficients $f_{R}^{\prime}(x, y)$ to $2 \times 2$ sub-block. Let $f_{R}^{\prime}(x, y)$ represents a $2 \times 2$ sub-block. Obtain the Gould coefficients from the $2 \times 2$ sub-block. Let the Gould coefficients $F_{r}^{\prime}(x, y)$ be represented by the equation

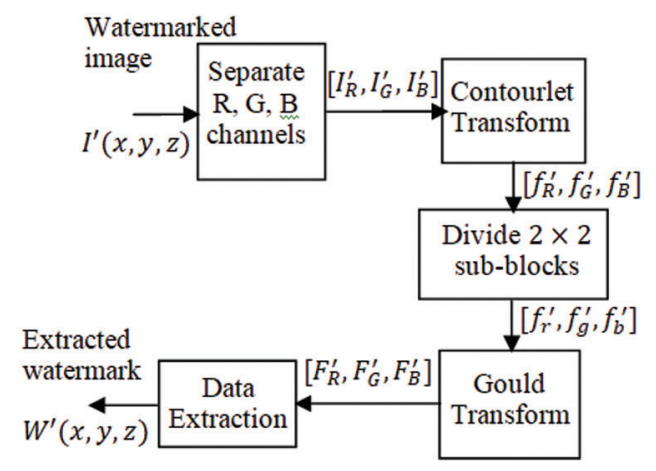

Figure 3: Process flow of proposed watermark extraction scheme

$F_{r}^{\prime}(x, y)=\left[F_{r}^{\prime}(1,1) F_{r}^{\prime}(1,2) F_{r}^{\prime}(2,1) F_{r}^{\prime}(2,2)\right]$

From the Gould coefficients $F_{r}^{\prime}(x, y)$ extract the watermark data $b_{i}^{\prime}$ using the equation.

$b_{i}^{\prime}=\left\{\begin{array}{ccc}1 & \text { if } & \frac{\alpha}{4} \leq \bmod \left(F_{r}^{\prime}(1,1), \alpha\right)<\frac{3 \alpha}{4} \\ 0 & \text { otherwise }\end{array}\right.$

The algorithm for watermark embedding is given below,

Input: Watermarked image $\mathrm{I}^{\prime}(\mathrm{x}, \mathrm{y}, \mathrm{z})$

Output: Extracted watermark $\mathrm{W}^{\prime}(\mathrm{x}, \mathrm{y}, \mathrm{z})$.

Step 1: Separate individual channels from the watermarked image $I^{\prime}(x, y, z)$.

Step 2: Obtain the Contourlet transform for the $\mathrm{R}$ channel $\mathrm{I}_{\mathrm{R}}^{\prime}(\mathrm{x}, \mathrm{y})$. Let the bandpass directional sub-band coefficients be $f_{R}^{\prime}(x, y)$

Step 3: Subdivided the bandpass directional sub-band Contourlet coefficients to $2 \times 2$ blocks. Let $\mathrm{f}_{\mathrm{r}}^{\prime}(\mathrm{x}, \mathrm{y})$ represents a sub-block.

Step 4: Obtain the Gould transform for the $2 \times 2$ subblocks $f_{r}^{\prime}(x, y)$ Let $F_{r}^{\prime}(x, y)$ be the Gould transformed coefficients.

Step 5: Extract the watermark data $b_{i}^{\prime}$ from the $2 \times 2$ subblocks $F_{r}^{\prime}(x, y)$ using Eq. (11).

Step 6: Repeat $2^{\text {nd }}$ step through $5^{\text {th }}$ step 5 to extract complete watermark data $b_{i}^{\prime}$. From the complete watermark data, reconstruct the watermark image $W^{\prime}(x, y, z)$. 


\section{Experimental Results}

MATLAB was used to implement the proposed technique with the four test RGB host images shown in Fig. 4. Each of these host images has a dimension of $512 \times 512$. The test watermark images are also RGB color images each having a size of $41 \times 41$ shown in Fig. 5 .

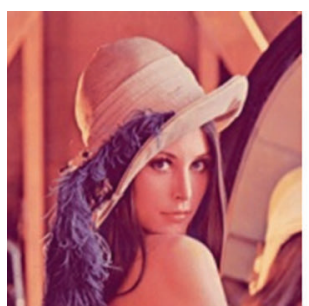

(a)

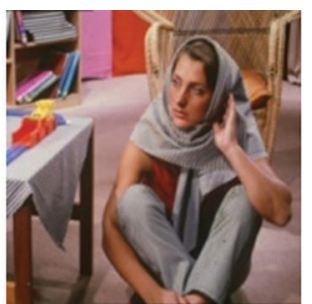

(b)

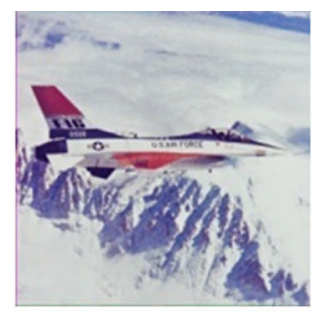

(c)

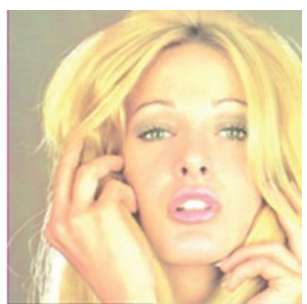

(d)

Figure 4: Sample host images (a) Lena (b) Barbara (c) Airplane (d) Tiffany

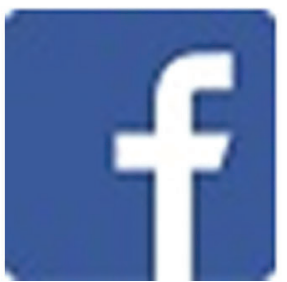

(a)

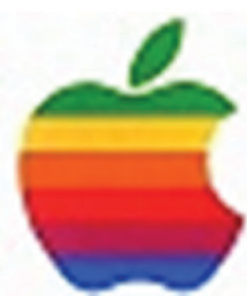

(b)

Figure 5: Watermark images (a) Facebook (b) Apple

The embedding rate can be calculated as,

bpp $=\frac{3 \times 41 \times 41 \times 8}{3 \times 512 \times 512}=0.0513$

Fig. 6 shows the watermarks extracted from different cover images without applying attacks. The robustness of the proposed hybrid watermarking algorithm was tested using the attacks listed in Tab. 1 and Fig. 7 shows the images that are subjected to such attacks.

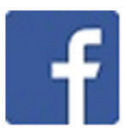

(a)

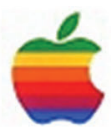

(b)

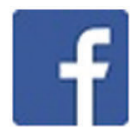

(c)

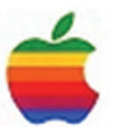

(d)

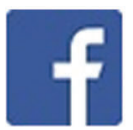

(e)

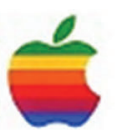

(f)

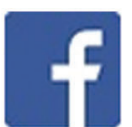

(g)

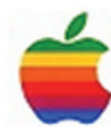

(h)

Figure 6: Extracted watermarks without attack from different host images (a, b) Lena (c, d) Barbara (e, f) Airplane (g, h) Tiffany

Figs. 8 and 9 displays the watermarks that have been extracted from the watermarked images that have been applied with attacks listed in Tab. 2. and Fig. 10 shows the watermarked images for the watermark 'Facebook' and 'Apple'. 
Table 1: Different types of attacks

\begin{tabular}{lll}
\hline Index & Attacks & Specification \\
\hline $\mathrm{a}$ & Salt and pepper noise & Noise density 0.05 \\
$\mathrm{~b}$ & Rotation & $45^{\circ}$ anticlockwise \\
$\mathrm{c}$ & Median filtering & Mask size $3 \times 3$ \\
$\mathrm{~d}$ & Cropping & $50 \%$ cropping \\
$\mathrm{e}$ & Brightening & Intensity increase by 60 \\
$\mathrm{f}$ & Darkening & Intensity decrease by 60 \\
$\mathrm{~g}$ & Gaussian low pass filtering & Filter size $3 \times 3$ \\
$\mathrm{~h}$ & Scaling & Scale by a factor 1.5 \\
\hline
\end{tabular}

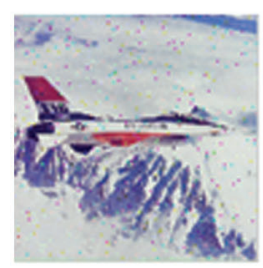

(a)

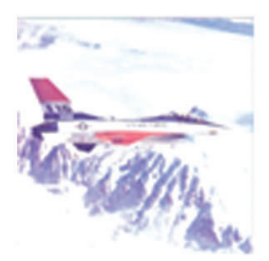

(e)

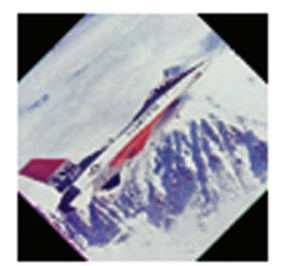

(b)

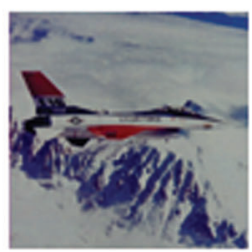

(f)

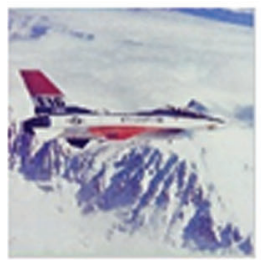

(c)

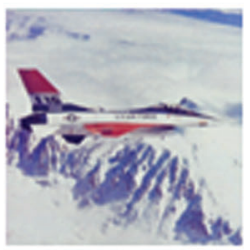

(g)

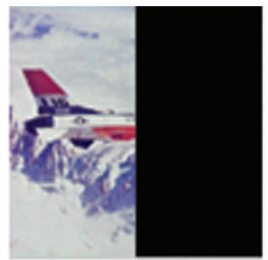

(d)

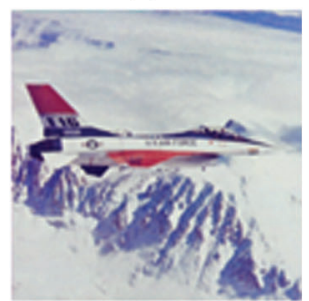

(h)

Figure 7: Different types of attacks (a) salt and pepper noise (b) rotation (c) median filtering (d) cropping (e) brightening (f) darkening (g) Gaussian low pass filtering (h) scaling

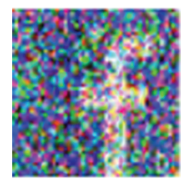

(a)

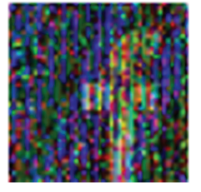

(e)

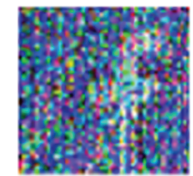

(b)

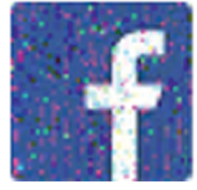

(f)

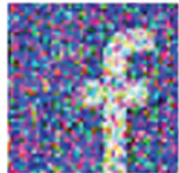

(c)

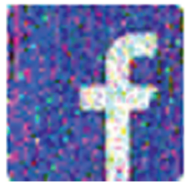

(g)

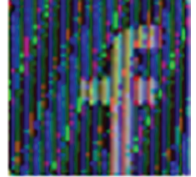

(d)

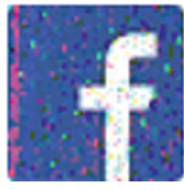

(h)

Figure 8: Extracted watermarks and their NCC values for the RGB host image airplane for different attacks (a) 0.8658 (b) 0.8467 (c) 0.8898 (d) 0.8322 (e) 0.7685 (f) 0.9829 (g) 0.9532 (h) 0.9804 


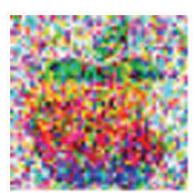

(a)

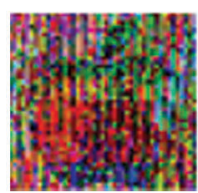

(e)

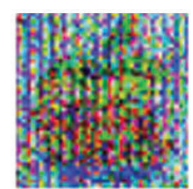

(b)

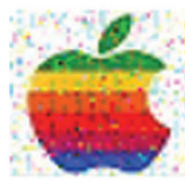

(f)

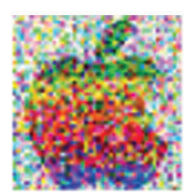

(c)

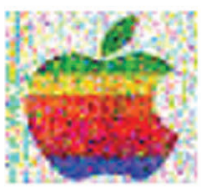

(g)

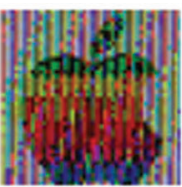

(d)

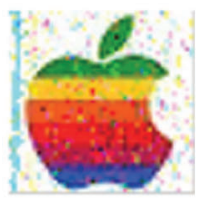

(h)

Figure 9: Extracted watermarks and their NCC values for the RGB host image airplane for different attacks (a) 0.9048 (b) 0.8427 (c) 0.9227 (d) 0.9096 (e) 0.8179 (f) 0.9918 (g) 0.9715 (h) 0.9897

Table 2: PSNR, SSIM and NCC for the proposed method

\begin{tabular}{llllll}
\hline \multirow{2}{*}{ Watermark } & Metrics & \multicolumn{4}{c}{ RGB Host image } \\
\cline { 3 - 6 } & & Airplane & Barbara & Lena & Tiffany \\
\hline Facebook & PSNR & 45.31 & 45.29 & 45.315 & 45.257 \\
& SSIM & 0.9954 & 0.9966 & 0.9961 & 0.9951 \\
\multirow{4}{*}{ Apple } & NCC & 0.9999 & 0.9999 & 1 & 0.9913 \\
& PSNR & 45.80 & 45.76 & 45.80 & 45.75 \\
& SSIM & 0.9951 & 0.9964 & 0.9959 & 0.9949 \\
& NCC & 1 & 1 & 0.99999 & 0.99516 \\
\hline
\end{tabular}

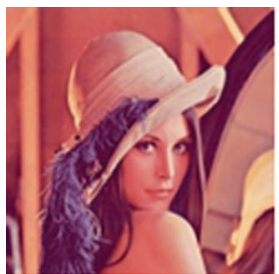

(a)

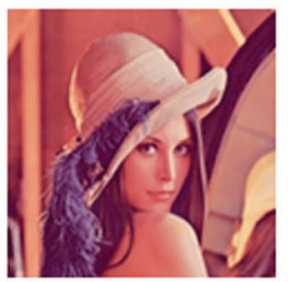

(e)

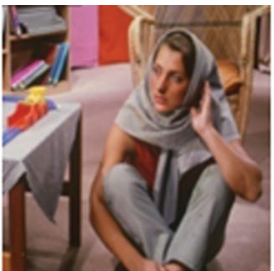

(b)

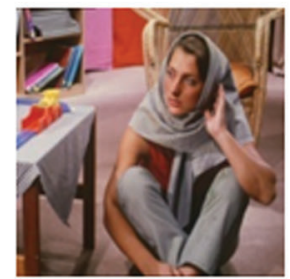

(f)

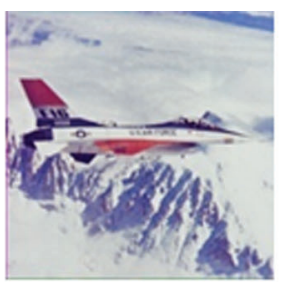

(c)

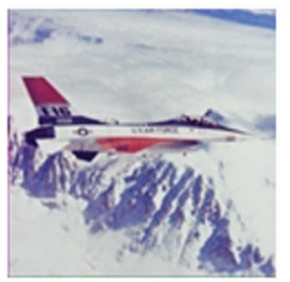

(g)

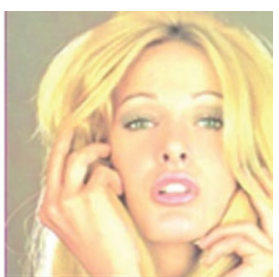

(d)

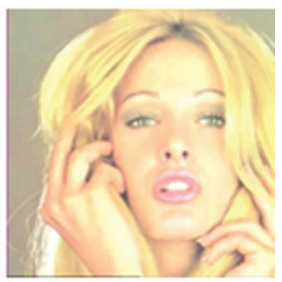

(h)

Figure 10: Watermarked images embedded with (a)-(d) Facebook, (e)-(h) Apple 
Tab. 2 shows the PSNR, SSIM, and NCC measurement for the proposed method. The average PSNR was found to be $45 \mathrm{~dB}$. The SSIM and NCC was found to be greater than 0.99. Fig. 11 shows the PSNR for varrying embedding capacity in multiple RGB host images. In all the Host images, the Apple watermark provides a higher PSNR than the Facebook watermark. The NCC (Normalized Cross Correlation) metric gives the robustness of the proposed method when subjected to various attacks. The NCC of the proposed method was compared with existing methods such as Improved Hybrid, Tensor decomposition and LU decomposition. Tab. 3 shows the NCC comparison and the proposed algorithm provides higher values than the existing methods.

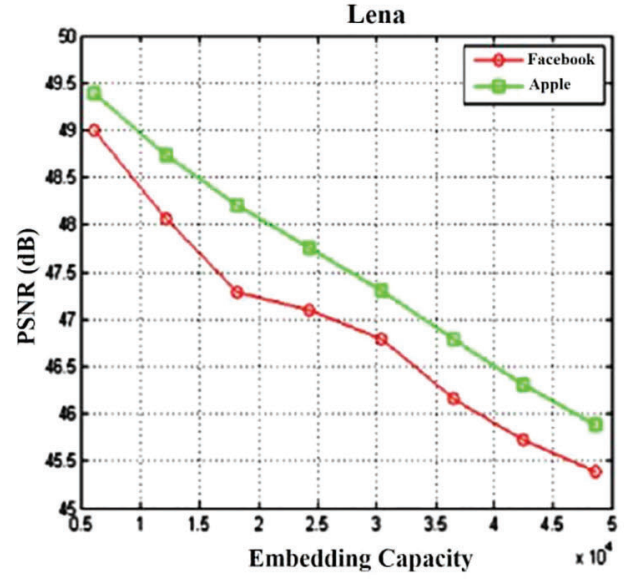

(a)

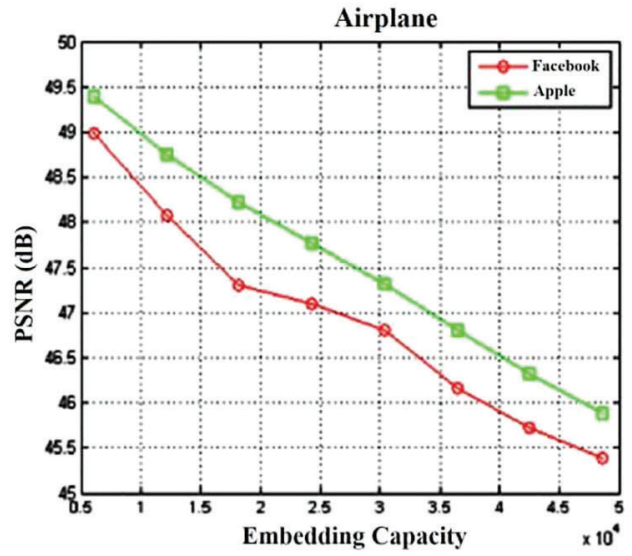

(c)

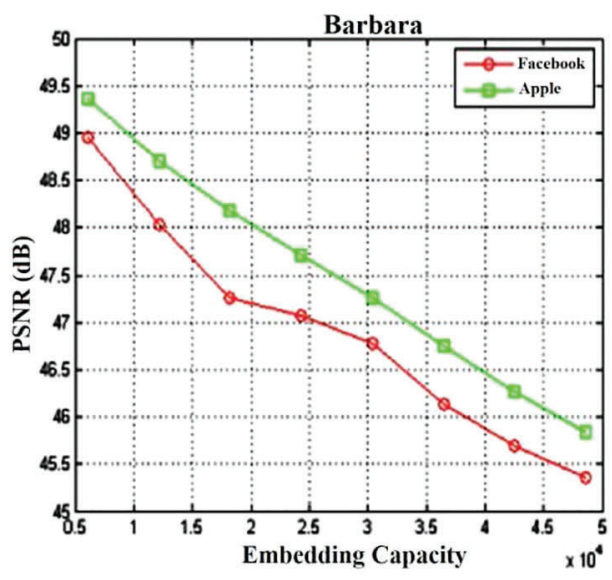

(b)

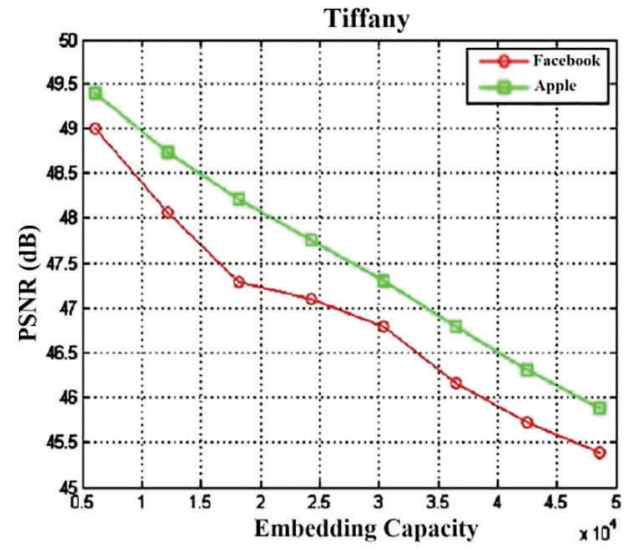

(d)

Figure 11: PSNR at Embedding rate for different RGB Host images (a) Lena (b) Barbara (c) Airplane (d) Tiffany 
Table 3: NCC comparison for the proposed and traditional methods

\begin{tabular}{lllll}
\hline Attack & Proposed & $\begin{array}{l}\text { Improved } \\
\text { hybrid }\end{array}$ & Tensor & LU \\
\hline Salt and pepper noise & 0.8853 & 0.8621 & 0.8522 & 0.8743 \\
Rotation & 0.8447 & 0.8742 & 0.8112 & 0.8331 \\
Median filtering & 0.9062 & 0.8891 & 0.8943 & 0.8962 \\
Cropping & 0.8709 & 0.8532 & 0.8542 & 0.8675 \\
Brightening & 0.7932 & 0.7821 & 0.8641 & 0.8043 \\
Darkening & 0.9873 & 0.9568 & 0.9682 & 0.9342 \\
Gaussian low pass filtering & 0.9623 & 0.9531 & 0.9523 & 0.9138 \\
Scaling & 0.9850 & 0.9631 & 0.9482 & 0.943 \\
\hline
\end{tabular}

\section{Conclusion}

This paper introduced a novel hybrid color image watermarking algorithm which uses Contourlet transform and Gould transform. The color image is initially transformed using Contourlet transform and the bandpass directional sub-band coefficients are again transformed using Gould transform. The secret data is induced on the $F_{11}$ coefficients of Gould. When compared to existing approaches, the generated watermarked image is exposed to various types of attacks, and the experimental findings indicate that the suggested watermarking algorithm is more resistant to attacks. The proposed algorithm provides an PSNR of around $45.25 \mathrm{~dB}$ and SSIM of 0.995 . The NCC obtained on the extracted watermark without attack is greater than 0.99. From the obtained results, it is concluded that the proposed method shows an outstanding performance than the traditional algorithms.

Acknowledgement: The author with a deep sense of gratitude would thank the supervisor for his guidance and constant support rendered during this research.

Funding Statement: The authors received no specific funding for this study.

Conflicts of Interest: The authors declare that they have no conflicts of interest to report regarding the present study.

\section{References}

[1] X. Zhu, J. Ding, H. Dong, K. Hu and X. Zhang, "Normalized correlation-based quantization modulation for robust watermarking," IEEE Transactions on Multimedia, vol. 16, no. 7, pp. 1888-1904, 2014.

[2] A. Khan, A. Siddiqa, S. Munib and S. A. Malik, "A recent survey of reversible watermarking techniques," Information Sciences, vol. 279, no. 1, pp. 251-272, 2014.

[3] M. Asikuzzaman and M. R. Pickering, "An overview of digital video watermarking," IEEE Transactions on Circuits and Systems for Video Technology, vol. 28, no. 9, pp. 2131-2153, 2017.

[4] M. Asikuzzaman, M. J. Alam, A. J. Lambert and M. R. Pickering, "Imperceptible and robust blind video watermarking using chrominance embedding: A set of approaches in the DT CWT domain," IEEE Transactions on Information Forensics and Security, vol. 9, no. 9, pp. 1502-1517, 2014.

[5] M. Asikuzzaman, M. J. Alam, A. J. Lambert and M. R. Pickering, "A blind and robust video watermarking scheme using chrominance embedding," in Proc. Int. Conf. on Digital Image Computing: Techniques and Applications (DICTA), Wollongong, NSW, Australia, pp. 1-6, 2014. 
[6] M. Kutter and S. Winkler, "A vision-based masking model for spread-spectrum image watermarking," IEEE Transactions on Image Processing, vol. 11, no. 1, pp. 16-25, 2002.

[7] B. Li, M. Wang, J. Huang and X. Li, "A new cost function for spatial image steganography," in Proc. Int. Conf. on Image Processing (ICIP), Paris, France, pp. 4206-4210, 2014.

[8] V. Sachnev, H. J. Kim, J. Nam, S. Suresh and Y. Q. Shi, "Reversible watermarking algorithm using sorting and prediction," IEEE Transactions on Circuits and Systems for Video Technology, vol. 19, no. 7, pp. 989-999, 2009.

[9] X. Li, B. Yang and T. Zeng, "Efficient reversible watermarking based on adaptive prediction-error expansion and pixel selection," IEEE Transactions on Image Processing, vol. 20, no. 12, pp. 3524-3533, 2011.

[10] C. H. Chou and K. C. Liu, "A perceptually tuned watermarking scheme for color images," IEEE Transactions on Image Processing, vol. 19, no. 11, pp. 2966-2982, 2010.

[11] G. Bhatnagar, Q. J. Wu and B. Raman, "Robust gray-scale logo watermarking in wavelet domain," Computers \& Electrical Engineering, vol. 38, no. 5, pp. 1164-1176, 2012.

[12] Z. Zhang and Y. L. Mo, "Embedding strategy of image watermarking in wavelet transform domain," in Proc. SPIE 4551, Image Compression and Encryption Technologies, Wuhan, China, vol. 4551, pp. 127-131, 2001.

[13] A. K. Singh, "Improved hybrid algorithm for robust and imperceptible multiple watermarking using digital images," Multimedia Tools and Applications, vol. 76, no. 6, pp. 8881-8900, 2017.

[14] Y. He, W. Liang, J. Liang and M. Pei, "Tensor decomposition-based color image watermarking," in Proc. Int. Conf. on Graphic and Image Processing (ICGIP), Hong Kong, China, vol. 9069, pp. 90690U, 2014.

[15] P. Tsai, Y. C. Hu and H. L. Yeh, "Reversible image hiding scheme using predictive coding and histogram shifting," Signal Processing, vol. 89, no. 6, pp. 1129-1143, 2009.

[16] J. Tian, "Reversible data embedding using a difference expansion," IEEE Transactions on Circuits and Systems for Video Technology, vol. 13, no. 8, pp. 890-896, 2003.

[17] B. Ou, X. Li, Y. Zhao, R. Ni and Y. Q. Shi, "Pairwise prediction-error expansion for efficient reversible data hiding," IEEE Transactions on Image Processing, vol. 22, no. 12, pp. 5010-5021, 2013.

[18] T. K. Tsui, X. P. Zhang and D. Androutsos, "Color image watermarking using multidimensional Fourier transforms," IEEE Transactions on Information Forensics and Security, vol. 3, no. 1, pp. 16-28, 2008.

[19] A. Mehto and N. Mehra, "Adaptive lossless medical image watermarking algorithm based on DCT \& DWT," Procedia Computer Science, vol. 78, no. 1, pp. 88-94, 2016.

[20] P. Bao and X. Ma, "Image adaptive watermarking using wavelet domain singular value decomposition," IEEE Transactions on Circuits and Systems for Video Technology, vol. 15, no. 1, pp. 96-102, 2005.

[21] I. A. Ansari, M. Pant and C. W. Ahn, "SVD based fragile watermarking scheme for tamper localization and selfrecovery," International Journal of Machine Learning and Cybernetics, vol. 7, no. 6, pp. 1225-1239, 2016.

[22] J. Li, C. Yu, B. B. Gupta and X. Ren, "Color image watermarking scheme based on quaternion Hadamard transform and Schur decomposition," Multimedia Tools and Applications, vol. 77, no. 4, pp. 4545-4561, 2018.

[23] Q. Su, G. Wang, X. Zhang, G. Lv and B. Chen, "A new algorithm of blind color image watermarking based on LU decomposition," Multidimensional Systems and Signal Processing, vol. 29, no. 3, pp. 1055-1074, 2018.

[24] Y. Lu and M. N. Do, "CRISP contourlets: A critically sampled directional multiresolution image representation," in Proc. SPIE 5207, Wavelets: Applications in Signal and Image Processing, San Diego, California, United States, vol. 5207, pp. 655-665, 2003.

[25] H. M. Le and M. Aburdene, "The discrete Gould transform and its applications," in Proc. SPIE 6064, Image Processing: Algorithms and Systems, Neural Networks, and Machine Learning, San Jose, California, United States, vol. 6064, pp. 60640I, 2006. 